\title{
Applying Process Mining and Semantic Reasoning for Process Model Customization in Healthcare
}

Silvana Pereira Detro ${ }^{\mathrm{a}, \mathrm{b} *}$, Eduardo Alves Portela Santos ${ }^{\mathrm{a}}$, Hervé Panetto ${ }^{\mathrm{b}}$, Eduardo Rocha Loures de Freitas ${ }^{\mathrm{a}}$ and Mario Lezoche ${ }^{\mathrm{b}}$

${ }^{a}$ Graduate Program in Production Engineering and Systems (PPGEPS), Pontificia Universidade Católica do Paraná (PUCPR), Curitiba, Paraná, Brazil; ${ }^{b}$ Université de Lorraine, CNRS, CRAN, Nancy, France

Silvana Pereira Detro

Email: sdetro@gmail.com

Pontifical University Catholic of Parana

1155, Imaculada Conceição

Prado Velho

80215-901 


\title{
Applying process mining and semantic reasoning for process model customization in healthcare
}

\author{
Process flexibility plays a key role in high variability environments, such as \\ healthcare. In this type of environment, the process model needs to change some \\ elements to adjust to specific sets of requirements. Thus, this paper proposes a \\ process model customizing method based on ontology and process mining. The \\ method proposed is applied in customizing process models for acute ischemic \\ stroke treatment. During process model customization, the method provides \\ decision-making support for users, thereby ensuring a structurally correct process \\ customization and enabling improves patient treatment by means of \\ recommendations.
}

Keywords: process model customization; ontology; semantic reasoning; process mining; healthcare

\section{Introduction}

The existence of different contexts is characteristic of some environments. For example, in the healthcare environments, patients require different types of treatment as a function of a number of factors, such as their specific characteristics, response to treatment, among other issues. Therefore, the process model needs to adapt to each existing context's specific environment.

Process models capable of changing to address different requirements are known as customizable (La Rosa et al., 2017) or configurable process models (Ayora et al., 2013a). In customizable process models, some of the process model elements, characterized by decision or variation points, can change (Torres et al., 2013; Ayora et al., 2013a). Therefore, each decision point refers to the selection of the changing elements in the process model. The selection of a given alternative in the decision point is driven by rules linked to them. Therefore, the process model (or process variant) is obtained through alternatives selected at each decision point, (La Rosa, Dumas e Ter Hofstede, 2009). 
The structurally and behaviorally correct variant must be guaranteed (i.e., all activities connected to enabling the execution of the process model present). In addition, requirements of the application context, regulations (internal and external), among other aspects, should be addressed (Valença et al., 2013; La Rosa et al., 2017, Van Der Aalst et al., 2008).

Therefore, many approaches for process model customization have been developed focusing on different aspects. The existing approaches lack recommendations in connection with the context of application provided to users when customizing the process model (La Rosa et al., 2017; Bühne, Halmans and Pohl, 2003, Valença et al., 2013). These recommendations would ensure that the process variant obtained respects the rules of the context, as well as enhancing the quality of the process by providing access to pertinent best practices, thus ensuring that the process variant is obtained according to each users' needs.

Providing recommendations is essential in an environment, such as the healthcare, where for a set of different factors different treatments may be available. In addition, considering the information about the patient's health provided by the physician, only the relevant options should be displayed to the user. Therefore, a method capable of providing options and recommendations regarding the patient's treatment can help to improve the quality of the treatment, and it can be used to avoid mistakes during the treatment, which can also reduce costs associate with the treatment.

Considering this context, this paper proposes a method to support decisionmaking during process model customization enabling achieving a process variant based on each users' needs and the requirements of the context. The method proposes to obtain a customizable process model through process mining, which allows to discover process variants, as well the rules to select them. Furthermore, the knowledge necessary to 
customize the process model is formalized in ontologies. Therefore, decision-making support for process model customization is provided through semantic reasoning.

Building the customized process model through process mining is a way to guarantee that the process model is capable of representing all application contexts. In addition, it allows to define the decision points and the requirements for selecting each alternative (Hallerbach, Bauer, \& Reichert, 2010a; Gottschalk et al., 2009). Process mining consists in analyzing event logs in order to obtain the process model, enabling deviations checks, process model improvements, etc. (Van Der Aalst et al., 2011). It also allows identifying the essential information to customize the process model as well as process model points where access to the information is relevant.

Process model customization may comprise a huge amount of information. Therefore, the knowledge required for customizing the process model is formalized in ontologies, which enable to share a common understanding of a specific domain. Semantic reasoning allows new knowledge, implied relationships, etc. to be derived (Andrew, 2004; Haav, 2004; Martinez-Gil, 2015; Obitko, 2007; Abburu, 2012).

In this way, the method for supporting the decision-making during process model customization consists basically in three elements: the customizable process model, ontologies and the questionnaire-model approach. One ontology contains the knowledge about the variation points. Another ontology contains the knowledge about the clinical guidelines, which is improved with the expert knowledge. These ontologies are merged into one ontology. The questionnaire is applied for obtaining the information about the patient's health. Then, this information is used for reasoning on the ontology, which shows the next activities available and provide recommendations about the treatment. As the user selects one of the available alternatives for the next activities, the process variant is built. In other words, a process model is created, and narrow it down during execution 
through the ontology reasoning based on the available (static patient information, guideline) or newly obtained information (e.g. questionnaire, lab results, among others).

When customizing a process model, the user may not be able to select the appropriate activities due the amount of information. Therefore, the proposed approach aims to fulfill this gap through a system for supporting the decision making, which provide recommendations about the process, and an overview about the next activities based on the information provided about the patient's health. This approach can help the physician to select the next steps of the patient's treatment, thus increasing the quality, as well decreasing errors and the related costs.

This paper proposes a case study of the approach proposed for acute ischemic stroke treatment. Healthcare is an environment with high process variability. Even the treatment for patients with the same diagnostic can vary due the many aspects involved, such as the symptoms displayed by the patient, the physician's knowledge set, resources available, etc. As result, several paths may be followed in establishing the patients' treatment. For this reason, decision-making support can help in avoiding mistakes during the treatment and guide users in making the proper decisions based on a number of factors.

This paper is structured as follows: Section 2 summarizes papers related to the topic; with the literature review discussed in Section 3, discussing concepts related to process variability, process mining techniques and ontologies. Section 4 outlines the approach proposed. The application of the approach proposed for customizing process models for acute ischemic stroke treatment is presented in this Section. Conclusions are drawn in Section 5.

\section{Related works}

The annotation of a process model with semantics has different focuses, such as semantic 
information retrieval (Luo et al., 2016; Li et al., 2014), cross-enterprise collaboration (Hoang, Jung and Tran, 2014), service governance (Cai et al., 2018), BPM systems interoperability (Rico et al., 2015), evaluation of the aspects related to the enterprise process flows (Ingvaldsen and Gulla, 2012). However, the customization of a process model by annotating the concepts an ontology is not addressed by many authors. In addition, the proposed approach is a step forward in the application of the semantics for process model customization by providing a decision support system in healthcare.

Process models can be customized in different ways. An analysis of the approaches applicable to customizing process models enable identifying gaps in process model customization, such as the lack of approaches capable of providing context related recommendations. Some approaches provide guidance for users, but only in connection with decision points (La Rosa et al., 2017). Therefore, any guidance targets guaranteeing that the process model customized is correct, but recommendations enable improving process quality and can help users to avoid mistakes in customizing the process model.

With respect to obtaining a customizable process model by means of process mining techniques, Li, Reichert and Wombacher (2008a) proposed to obtain a base process model and the respective customizable elements through a heuristic search algorithm. The results obtained from both the method proposed and the traditional process mining algorithms are then compared (Li, Reichert and Wombacher, 2008b). Later, the authors proposed to build the base process model from a clustering algorithm (Li, Reichert and Wombacher, 2010). Rozinat, Mans and van der Aalst (2006) proposed to establish decision points and properties that lead the cases to follow the same route through process mining algorithms.

A customizable process model can be obtained from a set of event logs as proposed by Buijs, Van Dongen and van der Aalst (2013). The first approach proposes 
obtaining the single process model by merging the event logs. In the second approach, a single process model describes the behavior of all event logs, and the event log is then individualized from this process model. The third approach proposes to discover a unique process model, in which each event log is used to configure the process model. The last approach proposes to combine the discovery and the process model configuration. Buijs and Reijers (2014) propose an alignment matrix for comparing the execution of the same process in different organizations. Within the organization, the comparison considers planned behavior as compared to actual behavior. The comparison with other organizations is made considering their design process variants.

Huang et al. (2013) propose process model customization by means of ontologies. One ontology formalizes knowledge in relation to variation points and another one formalizes the rules for the application context. An algorithm is proposed for process model configuration in which the rules and the configurable process model are the inputs and the process variant, the output. In customizing process models, requirements must be provided to a programmer engineer, resulting in an approach that is not user friendly. In this case, external regulations are not addressed and there is no guidance or recommendation during process model customization. El Faquih, Sbaï and Fredj (2014) propose a framework to enrich the customizable process model semantically. Later, the authors proposed the semantic validation of the configurable process models (El Faquih, Sbaï and Fredj, 2015).

Thus, there is a need for a customizable process model approach capable of providing recommendations about the context of application and capable of displaying for users how a choice made in one of the variation points impacts on the decisions in other variation points. These aspects can improve the customization of the process model (La Rosa et al., 2017; Bühne, Halmans and Pohl, 2003, Valença et al., 2013). Therefore, 
this paper aims to close these gaps by proposing a method for customizing the process model by means of ontologies. In addition, the method proposes to apply the questionnaire model approach, which guides users as they customize the process model according context-specific requirements. The next section discusses the relevant background for the development of the method proposed.

\section{Background}

The method proposed in this paper applies process mining to obtain the information needed to build a customizable process model. It also applies ontologies for customizing process models. In this way, this section discusses how to manage context variability, and how process mining and ontologies are applied in customizing process models.

\subsection{Process variability}

Process variability refers to obtaining a process model that represents a specific set of requirements in a domain (Reichert and Weber, 2012). Thus, some elements are common to all process variants, while other elements may be relevant only in specific process variants (Ayora et al., 2015). According Figure 1, there are two options when dealing with variability in a process model. One of these options, known as multi-model, refers to keeping the process variants separate in a repository. However, this may be expensive and inefficient, as each process variant must be developed from scratch (Ayora et al., 2013b; Hallerbach, Bauer and Reichert, 2010a).

The other option, known as single-model, refers to maintaining all process variants in a base process model. This option represents the common elements only once, thus facilitating model reuse (Ayora et al., 2013a). However, the resulting process model can be complex, difficult to understand, analyze, manage, and expensive to maintain (La Rosa et al., 2017). This option offers two options to customize process models known as 
customization by restriction or by extension. The concept of variation points is present in both options. Variation points correspond to the parts of the process model in which users must to select the next activity. A red circle represents variation points in Figure 1.

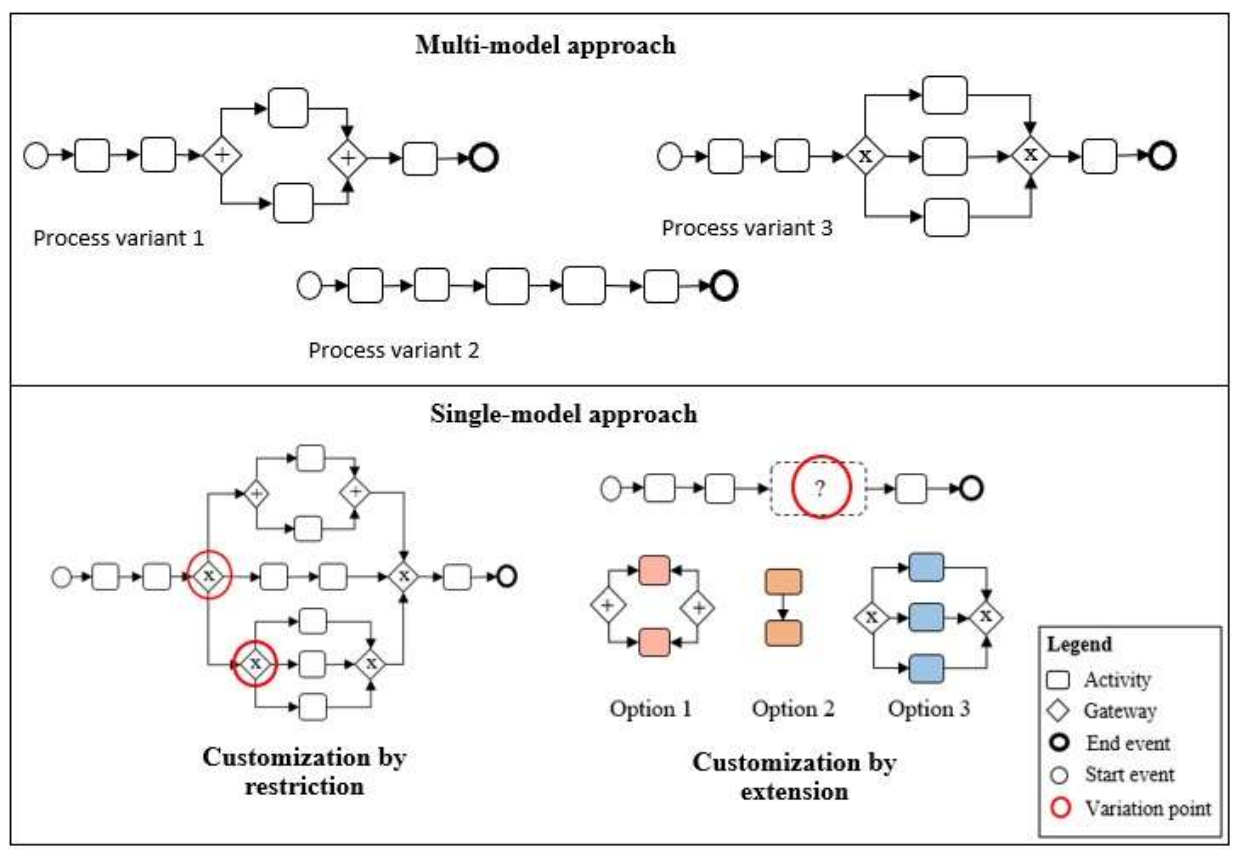

Figure 1. Approaches for process model variability

According to Figure 1, customization by restriction is performed when a single process model represents all process variants. Thus, a variant is obtained by selecting an alternative at each variation point, followed by removal of the elements that were not selected from the process model. However, when only the most common variant behaviors are represented in the base process model, customization by extension is performed. As result, the process variant is obtained by adding and/or modifying some elements in the base process model (La Rosa et al., 2017; Asadi et al., 2014). Therefore, there are three essential aspects in developing a customizable process model: the decision points, the alternatives for each decision point, and the requirements/rules defining their selection (Torres et al., 2013; Ayora et al., 2013a). 
Certain aspects must be addressed when building the customizable process model, such as the commonalities and differences among the process variants, as well the dependencies among the decision points. In addition, the resulting process variants must be structurally and behaviorally correct (Asadi et al., 2014, Van Der Aalst et al., 2008, La Rosa, 2009). Additionally, run-time flexibility and evolution of single variants must be ensured. Run-time flexibility refers to changes in the process model during run-time configurations. Evolution refers to adjusting process variants as a function of new specifications (Ayora et al., 2013a).

\subsection{Process mining}

Information systems are capable of recording information about process runs in the form of data logs. Therefore, data log analyses provide better understanding of the process model. One of these techniques, known as process mining promotes discovery of the process model, verifying process model compliance and enabling enhancements (Mans et al., 2013; Rozinat et al., 2009; Van Der Aalst and Dustdar, 2012).

The data log, known as event log, contains information about the cases, the activities performed, the time when the activity was performed (i.e., the timestamp) and who delivered the activity (the executor). In addition, the event log may contain additional information such as the patient's age, gender, among others (Jans et al., 2011; Van Der Aalst, 2012). Thus, process mining techniques provide in-depth understanding of what is happening inside organizations, which is the first step towards process enhancement (Weske, 2012; Günther et al., 2008).

Before the event log analysis, there is a pre-processing step, which is necessary to check log labels. The event log may contain different activities with the same label, or the same activities with different labels. For this reason, the concept of Semantic Business Process Mining appeared, combining BPM and semantic technologies (Pedrinaci and 
Domingue, 2007; De Medeiros et al., 2007). This concept refers to linking each element in the data log to a concept in the ontology, thus allowing new knowledge to be obtained through the inference engine (Detro et al., 2016).

\subsection{Ontologies and semantic reasoning}

Ontology can be defined as "an explicit specification of a concept definition", which means that the concepts in a domain and the existing relationships among them are formalized in ontologies (Gruber, 1995). Thus, the common understanding about a domain is formalized in ontologies, mostly for the purpose of applying, sharing and exchanging information (Gašević, Djuric and Devedžic, 2009). Ontologies also enable integration of knowledge by unifying Databases, Data Warehouses, and knowledge bases vocabularies (Djellali, 2013).

When building an ontology, knowledge representation and language should be taken into account. Selecting the language depends on what the ontology is meant to represent or its purpose (Sharman, Kishore and Ramesh, 2007; Taye, 2010). The languages for building ontologies are based on mark-up languages such as HTML (e.g., SHOE) and XML (e.g., SHOE, XOL, RDF, RDFS, OIL, DAML+OIL and OWL) (Corcho, Fernández-López \& Gómez-Pérez, 2007).

W3C (World Wide Web Consortium) recommends OWL as the standard semantic web ontology language for modelling ontologies (Song, Zacharewicz and Chen, 2013). This language allows the use of a reasoner, which enables new facts to be derived from ontologies, as well as allowing concepts that match specific definitions to be defined and performing ontology consistency checking. Thus, this language enables to derive knowledge, perform logical inferences, import and reuse different ontologies (Beimel and Peleg, 2011; Kalibatiene and Vasilecas, 2015; Menárguez-Tortosa and Fernández-Breis, 2013). Some techniques were developed to reuse ontologies, such as ontology mergers. 
Merging refers to unifying several ontologies into one by establishing correspondences among the ontologies (Pinto, Gómez-Pérez and Martins,1999; Noy and Musen, 2000).

Considering these aspects, this paper proposes a method that applies process mining in building a customizable process model and semantic reasoning for customizing the process model. Process mining allows identifying common elements among the variants, the elements that change, their changes, and the requirements for these changes to take place. Knowledge regarding aspects used to customize process models and the business context, including internal e external regulations, are formalized in ontologies. Then, semantic reasoning is used to provide users with recommendations for improving the process model.

\section{Method for customizing process variants}

The proposed method provides a decision-making support by means of recommendations for the rules applied in customizing the process model, and the regulations (internal and external) of the business context. Additionally, building the customizable process model through process mining promotes process model improvement through analyses of the actual process deliveries. Figure 2 presents the three steps of the method proposed, which consists in applying process mining techniques, the questionnaire template and ontologies. 


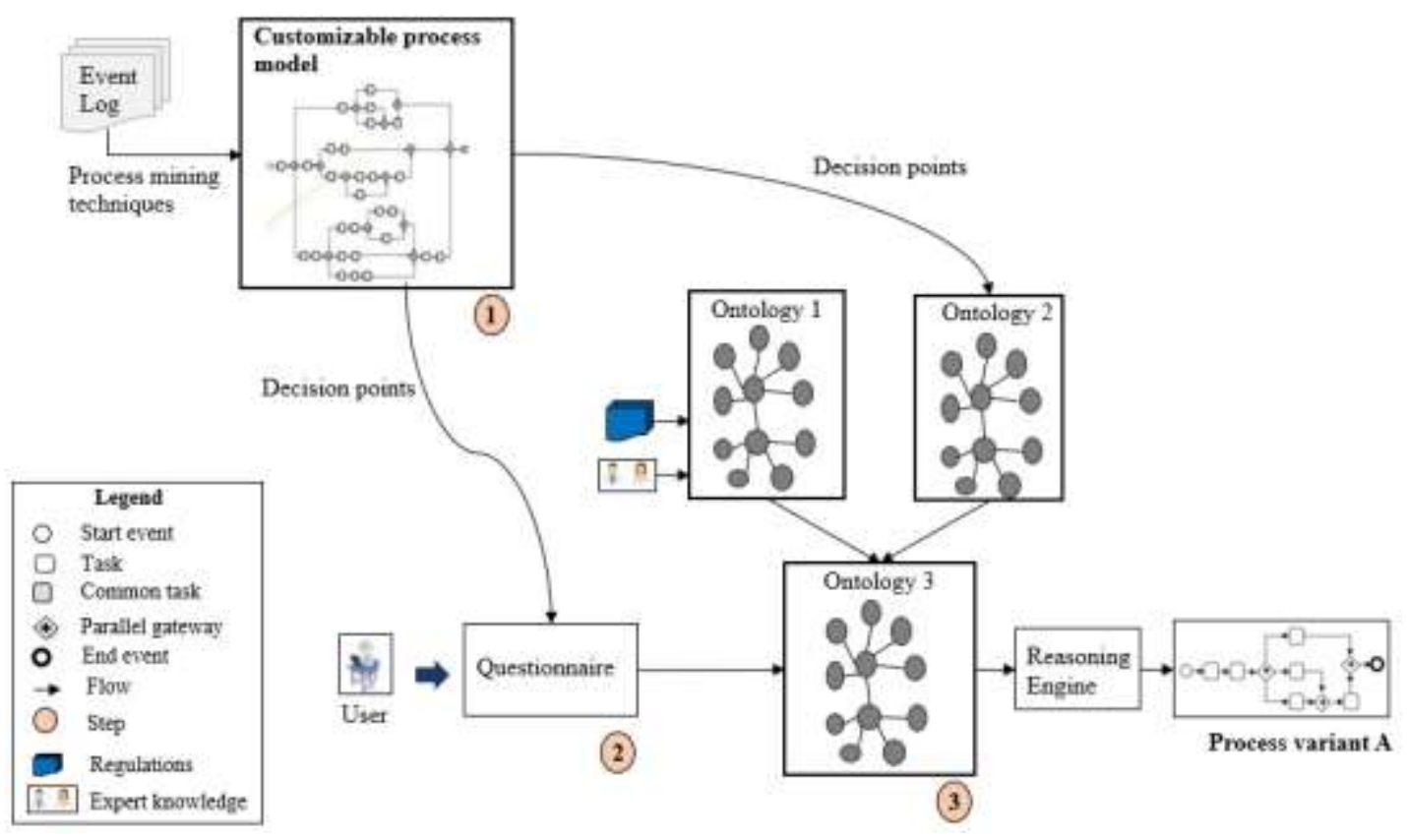

Figure 2. Approach for process model customization

According to Figure 2, process mining is the first step applied in building the customizable process model. This step enables identifying the elements that change, the decision points, the alternatives for each decision point, and the requirements attached to the alternatives. Identifying these aspects enables variant enhancements if necessary (Rozinat and Van Der Aalst, 2006b), thus improving the process model (Bose and Van Der Aalst, 2012; Huang et al., 2013).

By selecting an alternative at each variation point, the process model is customized. So, for the purpose of step 2 in customizing La Rosa's et al., (2009) questionnaire-model approach is proposed. This approach provides guidance for users during the configuration process by linking the decision points to questions and the alternative answers for the questions to the alternatives to be applied in the variation points. Therefore, the knowledge gathered in the first step about the aspects for customizing the process model is necessary in order to develop the questionnaire.

The rules that define the selection of the alternatives for each variation point may refer to internal and/or external regulations. As result, these rules may encompass a large 
amount of information. Therefore, step 3 proposes to build two ontologies for formalizing the necessary knowledge to the process model customization. The knowledge about the aspects related to the customization of the process model is formalized in one ontology. The knowledge about the internal and/or external regulations is formalized in the other ontology, which is enriched with the expert knowledge.

When the necessary knowledge for process model customization is formalized on the ontologies, both are merged into one ontology. As result, all the knowledge for customizing the process model is available in the new ontology. However, the reasoning relies on the information provided by the user through the questionnaire, which is developed when the rules for selecting the variation points and the recommendations are identified. Thus, when the user provides an information (or answers a question), the ontology reasoning happens, and the next steps and the related information are presented for the user.

Therefore, the proposed method can be used for providing support to the user, which can be a physician or a nurse. In this way, when the information about the patient is received, the semantic reasoning happens, and the activities and recommendations are proposed to the user. When the user selects an activity, the process model is customized. In addition, the user can visualize all the process model as it is customized. This method helps the user in selecting the appropriate treatment for the patient and facilitates the user to visualize each step and the information related with it.

Therefore, as the user answers the questionnaire and through the semantic reasoning, the user receives recommendations during the process model customization enabling to select the appropriate variant. Next sections present a case study for customizing the process model regarding the acute ischemic stroke treatment. 


\subsection{Case study description}

Acute ischemic stroke occurs when a part of the brain has the blood supply cut off, which can cause damage to the brain cells or even its death. In the last 15 years, the ischaemic heart disease and stroke are the principal causes of death in the world. Many patients survive to stroke, but many of them present some sequelae, which impacts the quality of life, the functional capacity and the health systems. Though, these impacts can be reduced by the early recognition the signs of stroke, admission by a specialized stroke unit, among others (World Stroke Organization, 2017).

According to Martins et al., (2012) there are four types of treatment for the acute ischemic stroke: intravenous thrombolysis protocol, protocol for intra-arterial thrombolysis, protocol for combined thrombolysis (intravenous and intra-arterial) and protocol for mechanical thrombolysis. The treatment selection is a complex decision, which relies on the patient's symptoms, the patient's medical history, the resources available at the moment, the internal and external rules, among others. Thus, the process model for the ischemic stroke treatment may contain several process variants and its selection is not a trivial task. Therefore, the method proposed in this paper provides support regarding the process model customization during the acute ischemic stroke treatment.

\subsection{Establishing the process model}

The first step corresponds to establishing the process model by analyzing the corresponding data logs. To this end, an event log was obtained from a Brazilian hospital for acute ischemic stroke treatment. The event log enabled identifying different types of information, such as the patient's personal information (age, gender, medical history), the exams performed during the treatment, time of onset of symptoms, time treatment started, 
among others. Due the complexity of the ischemic stroke treatment, focus was placed on one type of treatment, the clot burst drug administration, called intravenous recombinant tissue plasminogen activator (rt-PA) (Martins et al., 2012).

The information contained in the event log provides an understanding of the selection of the proper treatment. Despite, there still being missing information about the activities undertaken during the treatment delivered to patients. In this way, a complete process model cannot be obtained through event log analysis. Thus, a prescriptive process model is proposed for representing several scenarios considering several aspects in relation to the intravenous protocol treatment. The prescriptive process model contains the activities related to the clinical processes to deliver clinical services (i.e., diagnostic procedures) or clinical information (i.e., medical treatment record). Thus, it can be considered as a clinical workflow and it should be executed and running in an information system. Figure 3 indicates the prescriptive process model was built based on the event $\log$, the Brazilian guideline (Oliveira et al., 2012; Martins et al., 2012) developed for acute ischemic stroke treatment, and expert knowledge.

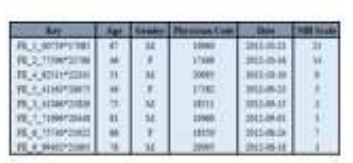

Event $\log$

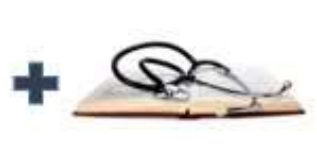

Clinical guideline

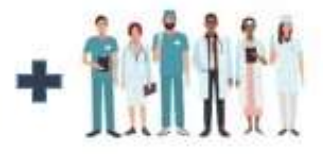

Physicians knowledge

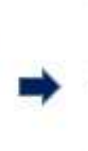

Figure 3. Aspects for developing the prescriptive process model

The clinical guideline shows how the treatment should be performed according to the patient's symptoms. However, the guideline addresses only the 'regular' ischemic stroke treatment related situations (Quaglini, 2008). Thus, expert knowledge can enhance the prescriptive process model by providing information that is not available in the 
medical guideline. Experts can also provide an understanding of the hospital rules and the sequence of activities during the treatment.

However, the clinical guidelines are recommendations about specific situations related to the patient's treatment. Thus, it is difficult to build the prescriptive process model from the clinical guideline and the expert knowledge. In this way, the process model obtained from the event log can be used to build the prescriptive process model by adding the knowledge obtained from the clinical guideline and the expert knowledge. In addition, the patient's medical information and the activities carried out during the patient's treatment were obtained from the event log, which shows the relation between the patient's symptoms and the treatment selected for the patient.

This approach for building the process model allows identifying possible improvements by comparing what is happening during the treatment (event log analysis) and what should happen (clinical guideline and expert knowledge). An excerpt of the prescriptive process model is depicted in Figure 4.

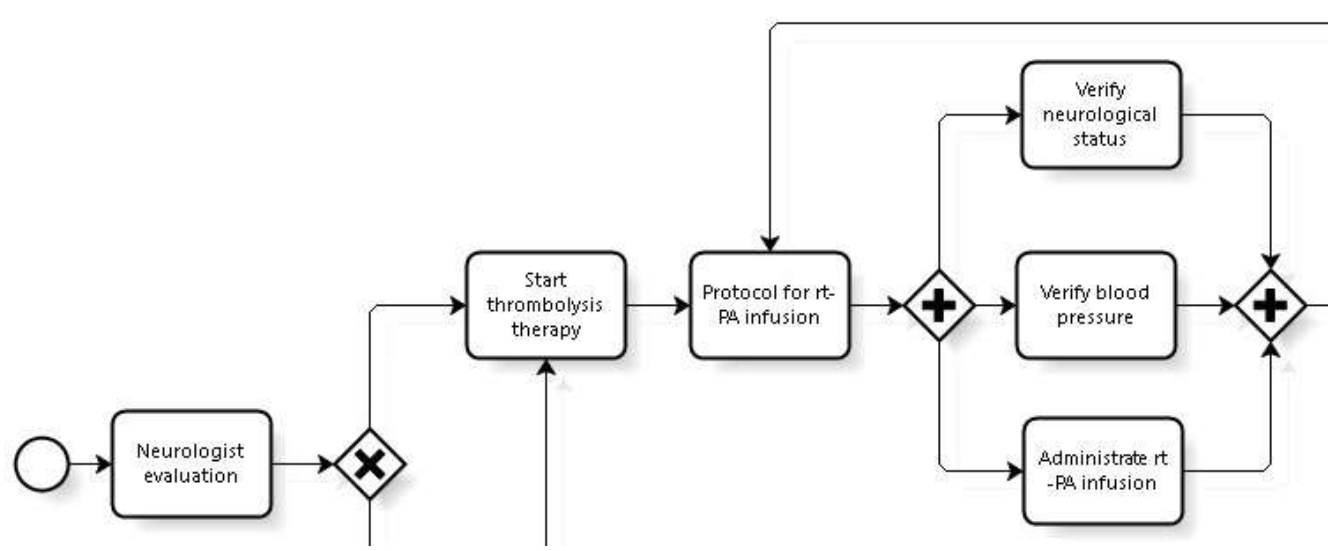

Figure 4 - Excerpt of the prescriptive process model

According to Figure 4, when the intravenous protocol treatment is selected, three activities are executed: thrombolytic therapy, check of the patient's neurological status, and of the patient's blood pressure (Martins et al., 2012). Thus, an event log related to the 
prescriptive process model is obtained, which allows simulating several possible scenarios, identifying the common elements among the variants, as well as any relations that may exist among them.

\subsubsection{Obtaining an event log}

An artificial event log can be obtained by means of Coloured Petri Net (CP-net or CPN) tools (De Medeiros and Günther, 2005). CPN is a language to model concurrent systems, which enables analyzing different scenarios, their properties and their results (Jensen, Kristensen and Wells, 2007; Aized, 2009). Based on this, a CP-Net was developed based on the prescriptive process model developed previously.

An excerpt of the developed Colored Petri Net is depicted in Figure 5, which contains places (ellipses or circles) marked with tokens, as shown in Figure 5 (place A2_4). Attached to each token there is a data value, called the token color. Therefore, the number of tokens and token colors on the individual places represent the state of the system. In addition, the CPN also contains transitions (rectangular boxes). Both transitions and places are connected by means of arcs.

Code segments (inscriptions) in CPN ML are attached to the transitions for supporting the creation of event logs, whenever the CP-net is executed (De Medeiros and Günther, 2005; Aized, 2009; CPN Tools, 2017). Results of individual CP-net simulation are files in ".cpnxml" format, exported through the ProM Import Framework to a single file in ".mxml" format. Files in this format can be analyzed by different tools that support several process mining algorithms, such as $\operatorname{ProM}^{1}$ (De Medeiros and Günther, 2005). The

\footnotetext{
${ }^{1}$ Process Mining framework. Process Mining Group, Math\&CS department, Eindhoven University of Technology, http://www.promtools.org
} 
CPN model simulation was run considering 1000 patients. By obtaining the event log, process mining can be applied allowing identifying the customizable process model and the related process variants.

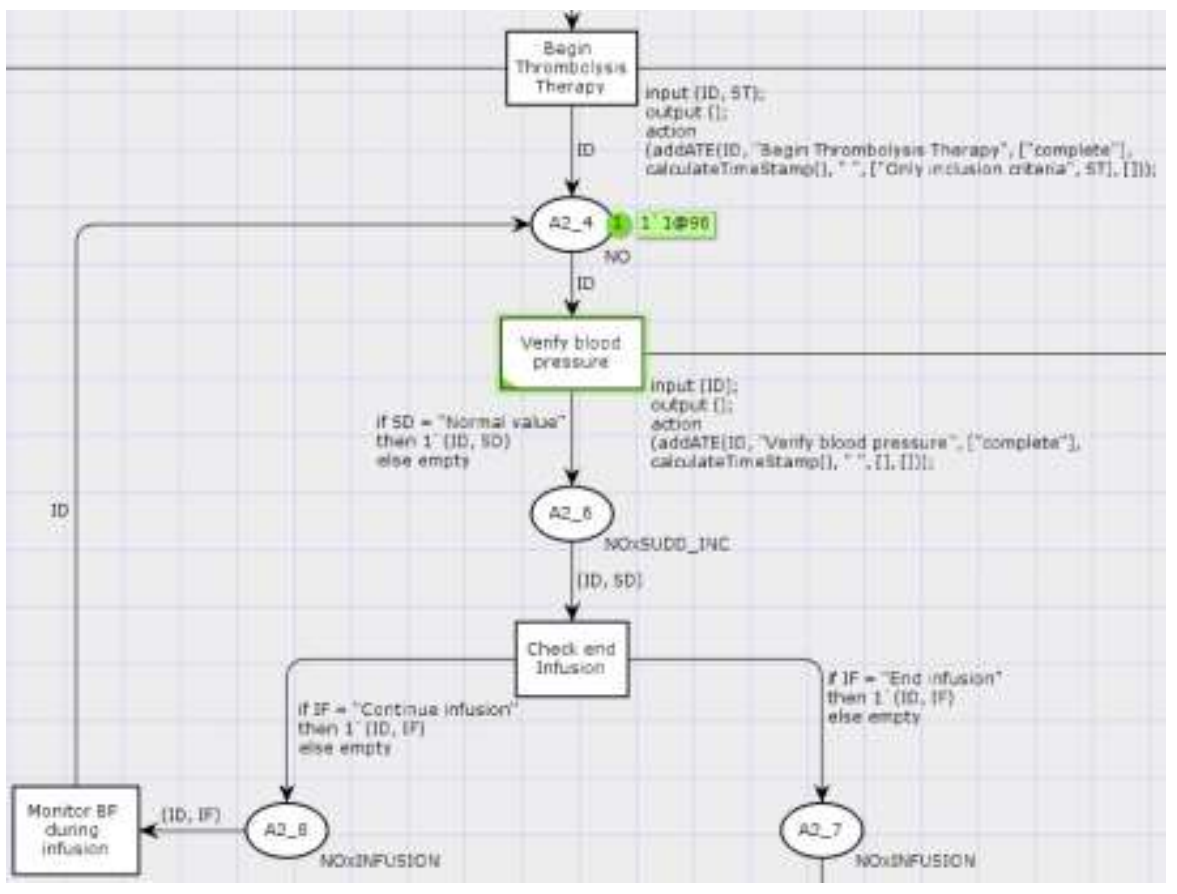

Figure 5 - Colored petri net

\subsubsection{Extracting process variants}

Process mining allows an event log to be examined under different perspectives. For example, the heuristic miner algorithm allows establishing decision points and the alternatives available for each one of them. Heuristic Miner is a discovery algorithm that infers and drafts direct graphs according to event frequency and sequence (FernándezLlatas et al., 2013). Figure 6 depicts the excerpt of the heuristic mining algorithm. 


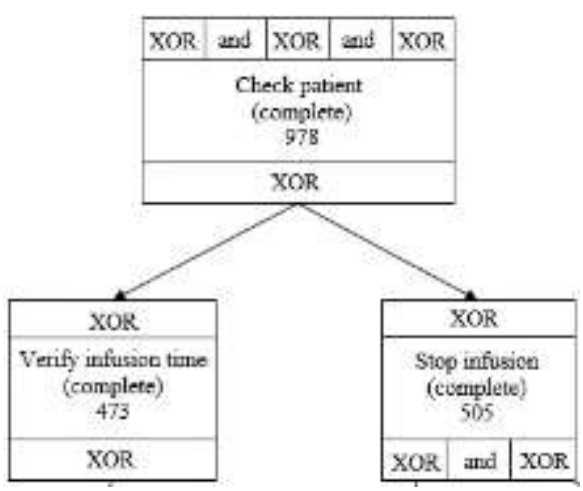

Figure 6 - Heuristic mining model for ischemic stroke treatment

Activity 'Check patient' in Figure 6 refers to one decision point for which the alternatives available are: 'Stop Infusion' or 'Verify infusion time'. However, this algorithm cannot be used to identify the rules for following one path instead of another. This way, decision-mining analysis can be applied. Decision mining allows discovering the properties of individual cases following the same path (Rozinat and Van Der Aalst, 2006b). As result, the decision miner can identify the three aspects for building a customizable process: the decision points, its alternatives and the rules attached to each alternative.

Decision mining is based on the decision tree algorithm $\mathrm{J} 48$, enabling pattern discovery and analysis in order to build classification models (Quinlan, 2014). Decision trees are composed of internal nodes, branches and leaf nodes. Each attribute test is represented by the respective internal node, branches represent test outcomes, and leaf nodes represent class labels. The root node is the topmost node of the tree. Therefore, a rule is found when following a path from the root to a leaf node (Agrawal and Gupta, 2013). Figure 7 shows the decision tree related to activity 'Select Treatment'. 


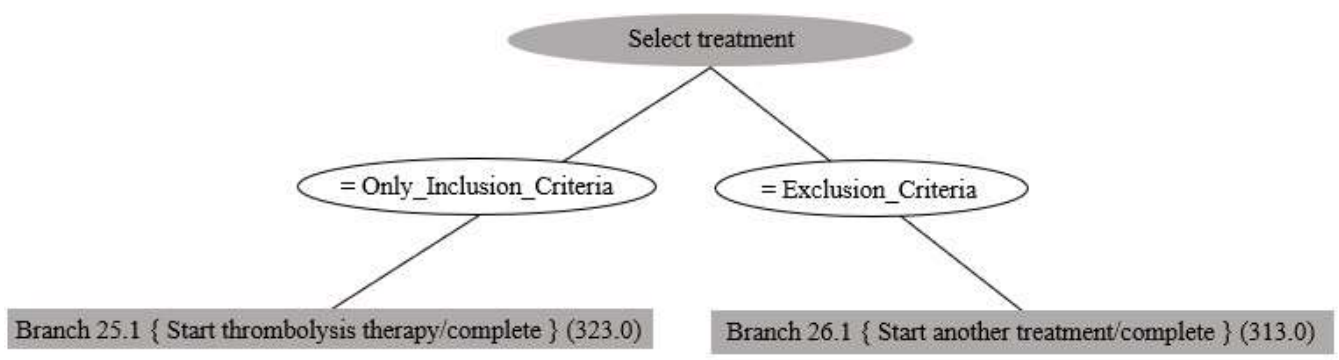

Figure 7 - Decision tree for the variation point 'Check patient'

According to the decision tree in Figure 7, if the patient only displays symptoms defined as inclusion criteria in the clinical guidelines for ischemic stroke, thrombolysis therapy is selected. However, if the patient displays any symptoms defined as exclusion criteria, then thrombolysis therapy cannot be performed, and another treatment must be selected (Martins et al., 2012). In this case, the decision miner algorithm allowed discovery of nine decision points, their alternatives and the rules for selecting the available alternatives. Table 1 summarizes these aspects.

Table 1 - Decision points, alternatives, and rules attached

\begin{tabular}{|c|c|c|}
\hline Decision points & Alternatives & Rules \\
\hline \multirow{2}{*}{ Select treatment } & Start thrombolysis therapy & Only inclusion criteria \\
\hline & Start another treatment & Exclusion and/or inclusion criteria \\
\hline \multirow{2}{*}{ Check patient } & Verify end of infusion & Haemorrhagic complications \\
\hline & Stop infusion & Without haemorrhagic complications \\
\hline \multirow{2}{*}{ Verify end of infusion } & Finalize rt-Pa infusion & Time of infusion $>=90$ minutes \\
\hline & Infusion treatment & Time of infusion $<90$ minutes \\
\hline \multirow{3}{*}{$\begin{array}{l}\text { Manage } \quad \text { BP } \quad \text { (blood } \\
\text { pressure) }\end{array}$} & Monitor changes in blood pressure & Normal blood pressure \\
\hline & $\begin{array}{l}\text { Verify if patient has contra- } \\
\text { indication of bb (beta blocker) }\end{array}$ & Patient with hypertension \\
\hline & $\begin{array}{l}\text { Provide fluid replacement or } \\
\text { vasoactive agents }\end{array}$ & Patient with hypotension \\
\hline \multirow{2}{*}{$\begin{array}{l}\text { Verify if patient has } \\
\text { contra-indication of bb }\end{array}$} & $\begin{array}{lll}\text { Administrate } & \text { Metoprolol or } \\
\text { Esmolol } & & \\
\end{array}$ & Patient has contra indication of $b b$ \\
\hline & $\begin{array}{ll}\text { Administrate } & \text { Sodium } \\
\text { Nitroprusside } & \\
\end{array}$ & Patient has not contra indication of $b b$ \\
\hline \multirow{2}{*}{ Verify CT scan results } & Restarting infusion & No intracranial haemorrhage \\
\hline & Discontinue infusion & Intracranial haemorrhage \\
\hline
\end{tabular}




\begin{tabular}{|c|c|c|}
\hline \multirow{4}{*}{ Verify lab results } & Provide red cell & Abnormal haematocrit \\
\hline & Provide platelets & Low platelet count \\
\hline & Repeat cryo & Low fibrinogen \\
\hline & Provide fresh plasma & Abnormal PT or aPTT \\
\hline \multirow{2}{*}{ Discontinue infusion } & Monitor neurological status & $\begin{array}{l}\text { No evidence of bleeding in the central } \\
\text { nervous system }\end{array}$ \\
\hline & $\begin{array}{l}\text { Request a haematology and a } \\
\text { neurosurgery appointment }\end{array}$ & $\begin{array}{l}\text { Evidence of bleeding in the central } \\
\text { nervous system }\end{array}$ \\
\hline \multirow{2}{*}{$\begin{array}{l}\text { Monitor changes in } \\
\text { neuro status }\end{array}$} & Provide blood products & $\begin{array}{l}\text { Clinical status deterioration after } 4 \text { to } 6 \\
\text { hours }\end{array}$ \\
\hline & Monitor patient & No deterioration of the clinical status \\
\hline
\end{tabular}

Table 1 presents the necessary information to customize the process model and essential for the questionnaire development. The dependency between the decision points can also be identified through the decision miner algorithm, as depicted in Figure 8.

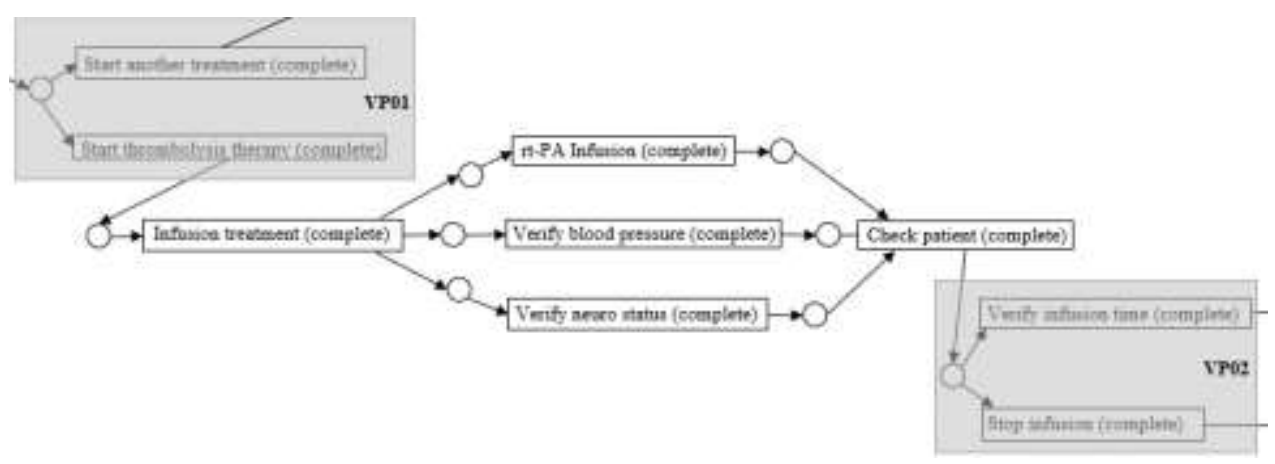

Figure 8 - Dependence among variation points

According to Figure 8, the first variation point (VP01) refers to selecting the treatment for the patient. The Figure also shows that the second variation point (VP02) refers to patient follow up during the infusion. Thus, variation point VP02 is enabled only when the therapy selected in variation point VP01 is thrombolysis. Figure 9 shows the relations among the decision points. 


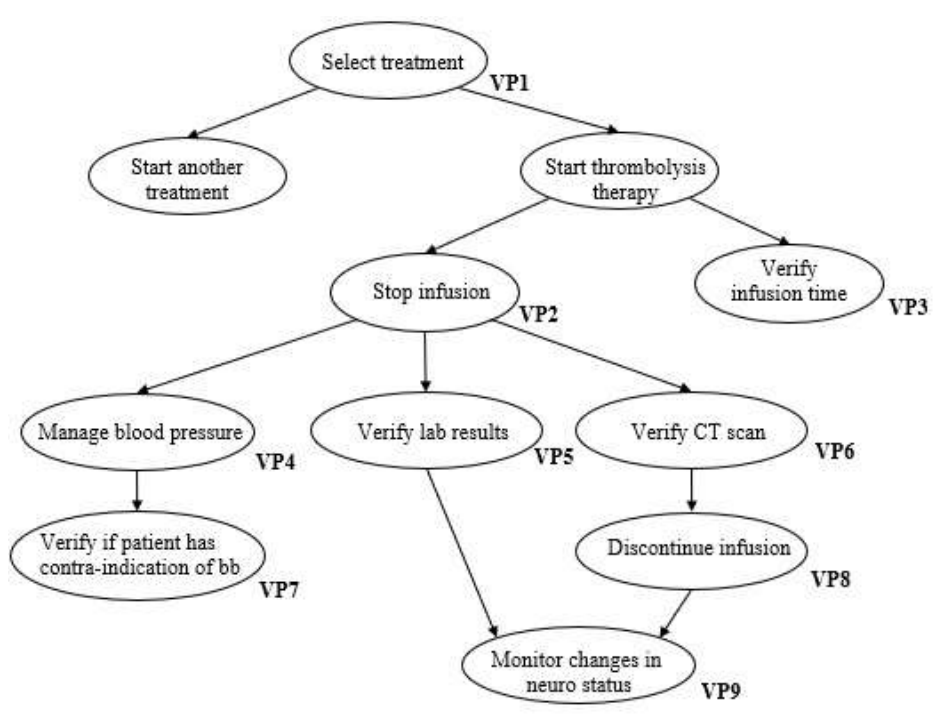

Figure 9 - Relations among variations points

According to Figure 9, two types of variation points may be identified. The first type, known as mandatory variation point, refers to selecting the process variant. For example, decision point VP01 is in reference to selecting the treatment. The second type is called optional variation point and is in reference to the execution of specific activities. For example, variation point VP05 refers to checking lab results. As can be seen in Figure 9, the optional variation points are inherited by the variation point defined as mandatory. Therefore, when a mandatory variation point is selected, this enables the related optional variation points (Bühne, Halmans and Pohl, 2003).

Understanding the interdependencies among the decision points enables to define the order of the questions. This information (Figure 9) and the information related to the aspects for process model customization (Table 1) are useful in defining the recommendations that should be provided to users during process model customization and the process model points for which each recommendation is relevant. This information is essential in the next steps with respect to the development of the questionnaire and the ontology based on process model customizing aspects. 


\subsection{The questionnaire-model development approach}

The selection of an alternative in the variation points depends on rules. These rules refer to specific application context requirements. Thus, users provide the requirements necessary to customize the process model. In this way, step 2 shown in Figure 2, refers to the development of the questionnaire-model approach for process model customization. In this approach, the variation points are connected to the respective questions, whose answers refer to the alternatives available for the different variation points. Therefore, as users select an alternative for a question, the other alternatives are disabled. As result, the process model is customized as users answer the questionnaire.

The questionnaire-model approach allows facts to be defined as mandatory or non-mandatory, i.e., facts that must be input by users or facts that can be set applying default values (Hallerbach, Bauer and Reichert, 2010b). In addition, it enables defining the order of dependence between facts and questions (see La Rosa et al., 2009). The dependence between the variation points (in Figure 9) establishes the order in which the questions should be asked to users. So, the decision miner provides the information necessary to develop the questionnaire as depicted in Figure 10.

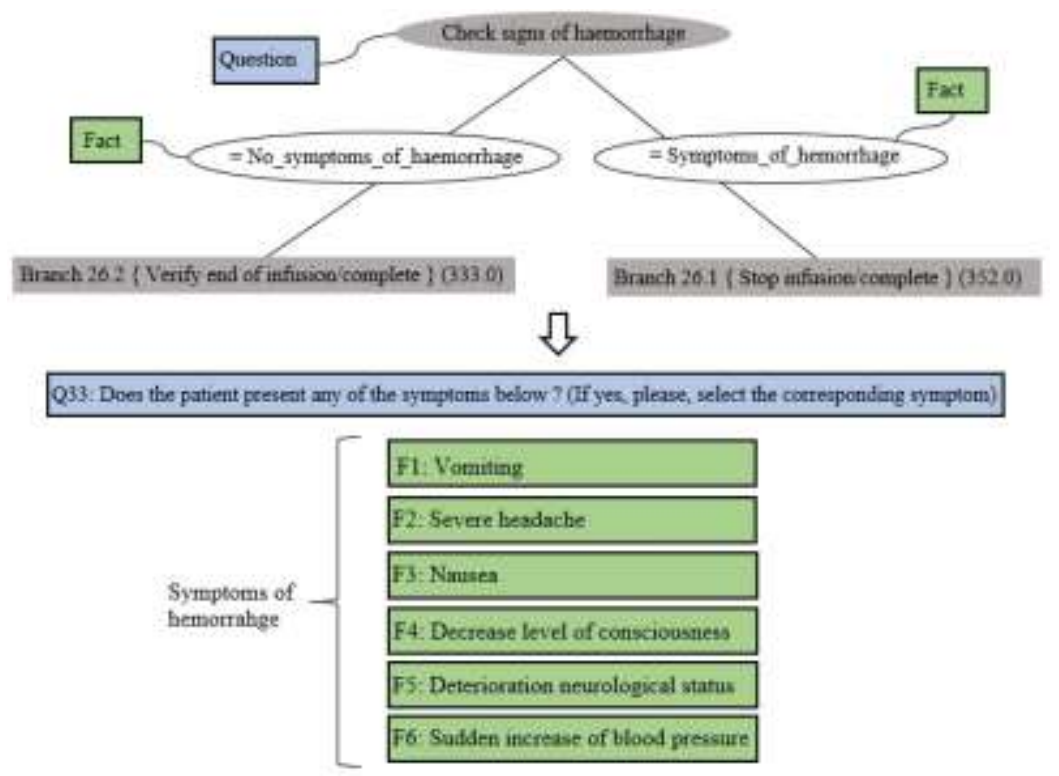

Figure 10 - Using a decision tree to develop the questionnaire-model approach 
Figure 10 shows that decision tree root and branches refer respectively to a question and the domain facts in the questionnaire. Therefore, the questionnaire was built considering the information related to the decision points, its alternatives, the rules linked (Table 1) and the information gathered from the clinical guideline for ischemic stroke. Following the development of the questionnaire, the ontologies can then be developed.

\subsection{Semantic reasoning for customizing the process model}

This paper does not intend to build ontologies, but rather focus on deploying ontologies. As depicted in the approach shown in Figure 2, two ontologies need to be developed. According to Figure 11, one ontology refers to the requirements (internal and/or external) in connection with the application context and enhanced with expert knowledge. The second ontology refers to the knowledge related to the decision points, their alternatives and the rules linked to them. After this, a new ontology is obtained by merging both ontologies.

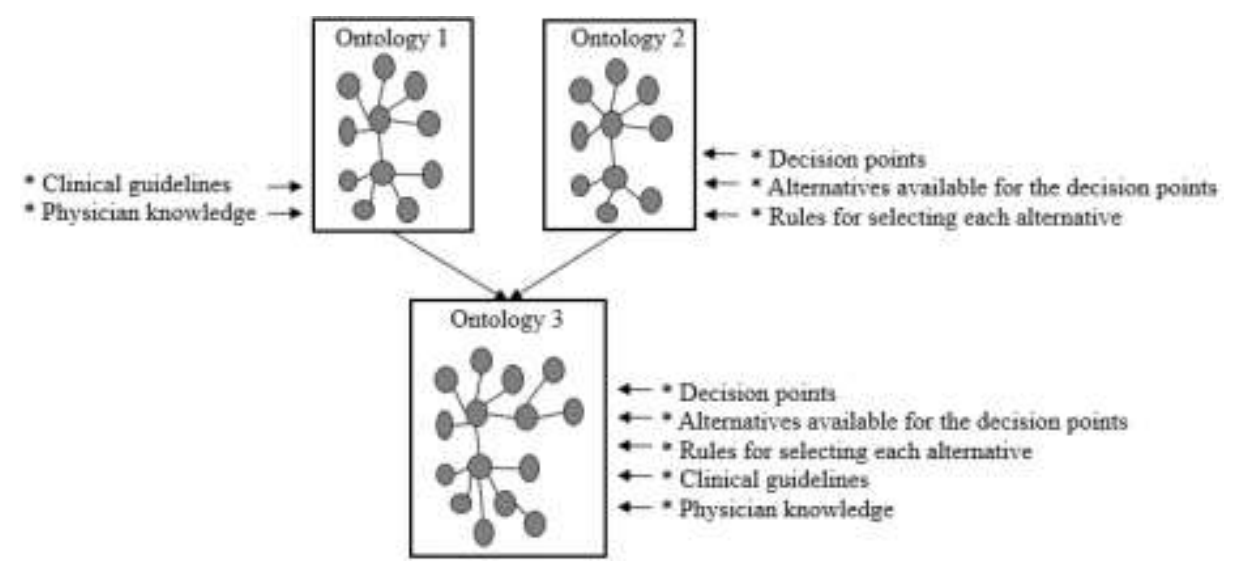

Figure 11 - Ontologies for process model customization 
The process model ontology is developed considering the dependence among the variation points (Figure 7) and the information about the variation points (Table 1) as follows:

Table 2 - Using the knowledge from the decision tree to build an ontology

\begin{tabular}{|l|l|}
\hline Elements in the Decision Tree & Elements in the Ontology \\
\hline Branch & Data properties \\
\hline Leaf node & Classes \\
\hline
\end{tabular}

Table 2 shows that the decision tree leaf nodes and branches refers, respectively, to the classes and the data properties in the ontology. The data properties represent the requirements of the application context and are set as 'true' or 'false' by the user. Figure 12 shows the relations between variation point 'Check patient', which was discovered through the decision mining algorithm and the ontology related with the respective variation points. Figure 7 shows the decision tree of this variation point.

Figure 12 (a) shows the variation point 'Select treatment', its alternatives and the rules for selecting each alternative. If the patient displays only inclusion criteria, thrombolysis therapy is selected. However, if the patient displays any exclusion criteria, another treatment must be selected. This information is necessary in order to build the ontology for the variation points, as shown in Figure 12 (b). The ontology contains a class named 'VP01_Select_Treatment', which refers to the variation point 'Select treatment' (Figure 12 (a)). This class has two subclasses, which refer to the alternatives available for this variation point called 'VP01-1_Start_Another_Treatment' and 'VP012_Start_Thrombolysis_Therapy'. The requirements for selecting the alternatives in Figure 12(a) called 'Only inclusion criteria' and 'Exclusion criteria' are defined as properties in the ontology (Figure 12(c)). 


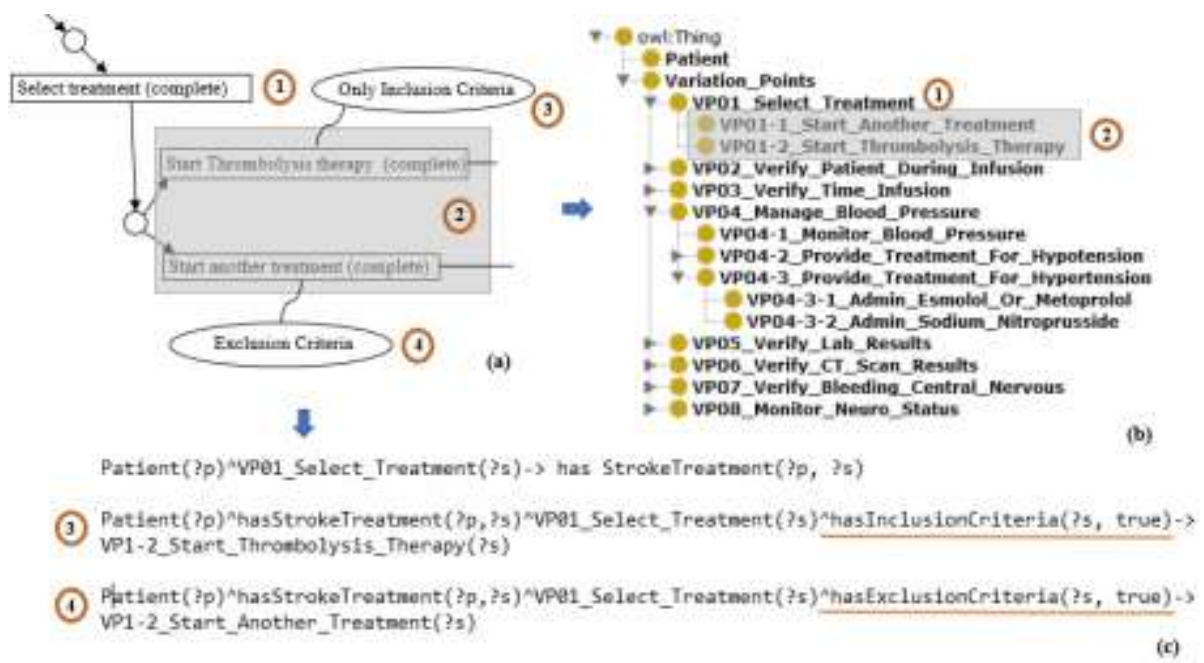

Figure 12 - Relations between the decision points and the ontology about the decision points

The rules for providing users with recommendations are based on the requirements for selecting each alternative (Table 1) and the dependence between the variation points (Figure 9). Thus, as users select the alternative for a given variation point, one or more variation points are enabled and displayed. For example, Figure 12(c) shows SWRL rules, stating that thrombolysis therapy is selected when users mark data property 'hasInclusionCriteria' as being True. But, if users set data property 'hasExclusionCriteria' as True, then another treatment is selected. Figure 13 shows the ontology before and after the logic step.
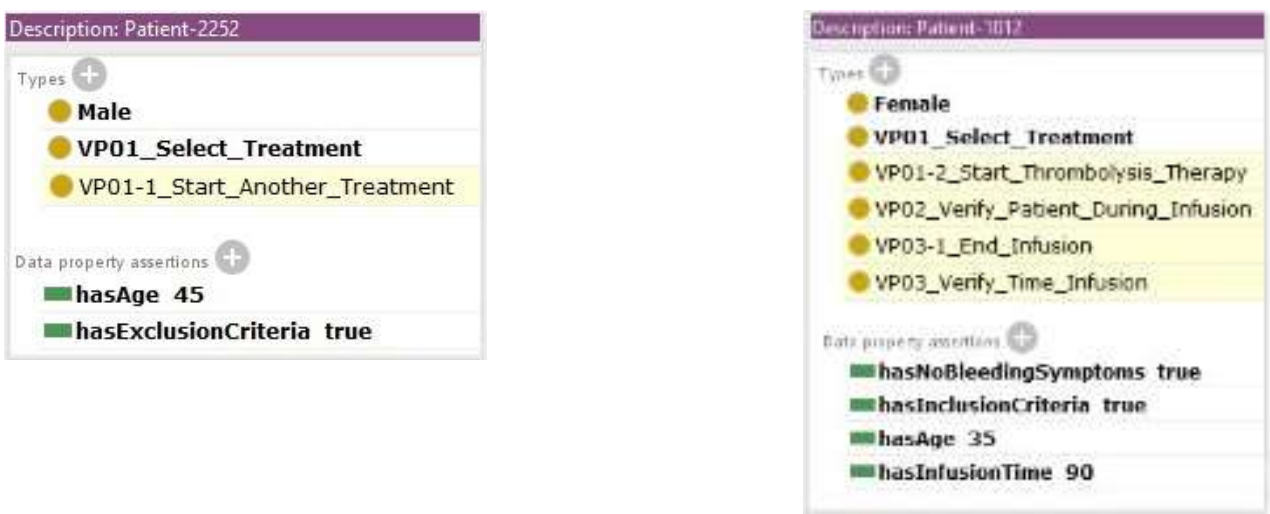

Figure 13 - Ontology before and after the logic step 
Figure 13(a) shows that data property 'hasExclusionCriteria' was set as true since the patient (ID 2252) displays symptoms classified as exclusion criteria. As result, the logic step shows the selection of another treatment for this patient. On the other side, Figure 13(b) refers to the treatment provided for a patient (ID 1012) that only displays inclusion criteria. Therefore, data property 'hasInclusionCriteria' is set as true, resulting in the selection of the thrombolytic therapy. This ontology also shows users the next variation point (VP02_Verify_Patient_During_Infusion), enabled by the selection of rtPA infusion. This variation point is in connection with checking the patient's neurological status. In case of signs of bleeding, the infusion is interrupted. Otherwise, the nurse or physician should check the infusion time, which cannot exceed 90 minutes. The other ontology formalizes the knowledge as regards the Brazilian medical guideline for acute ischemic stroke. Thus, through this ontology's rationale, users receive recommendations about the best practices for the patient's treatment, as depicted in Figure 14 .

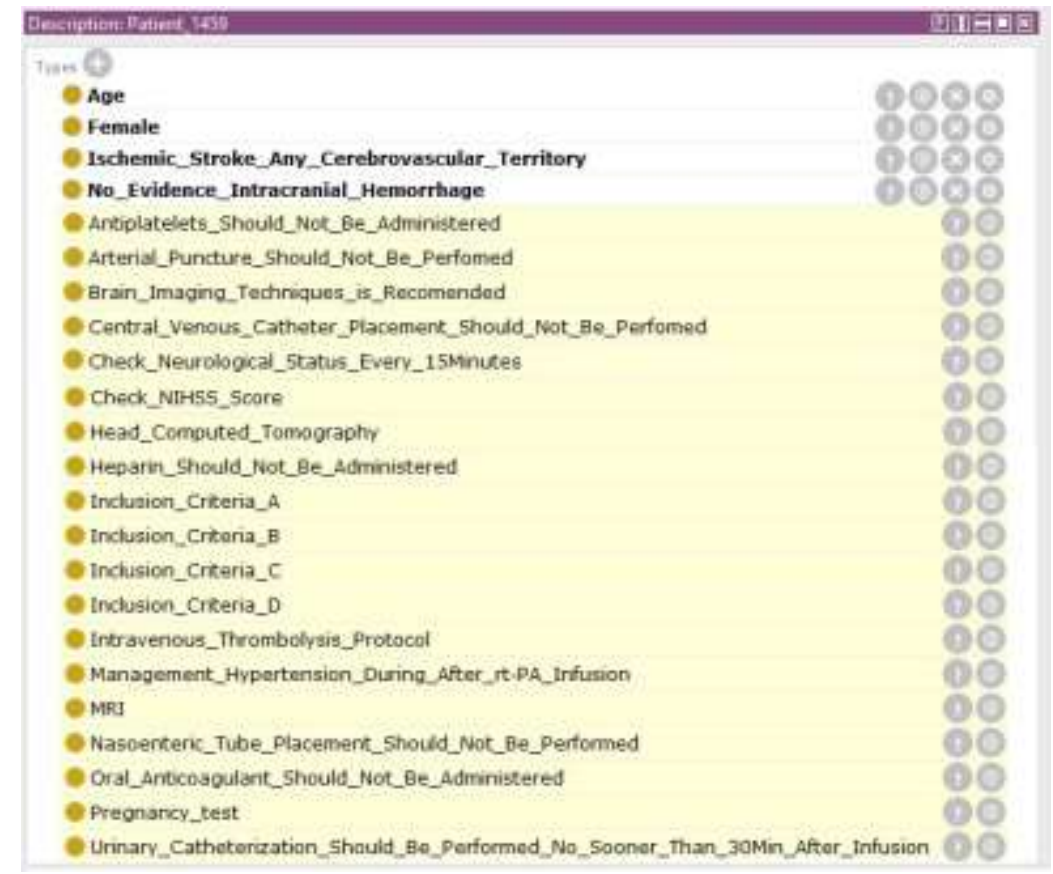

Figure 14 - Recommendations on the patient's treatment 
Then, both ontologies were merged in Protégé. Merging ontologies means unifying ontologies into a single one. Usually, the ontology merged deals with the same subject, but the level of generality can change (Pinto, Gómez-Pérez and Martins, 1999). After merging the ontologies, correspondences among the ontologies should be established and there should be an inconsistency check. In this case, there may a level of overlap among some concepts, others may have different names or structure and concepts but the same meaning (Noy and Musen, 2000). Figure 15 depicts the resulting ontology.

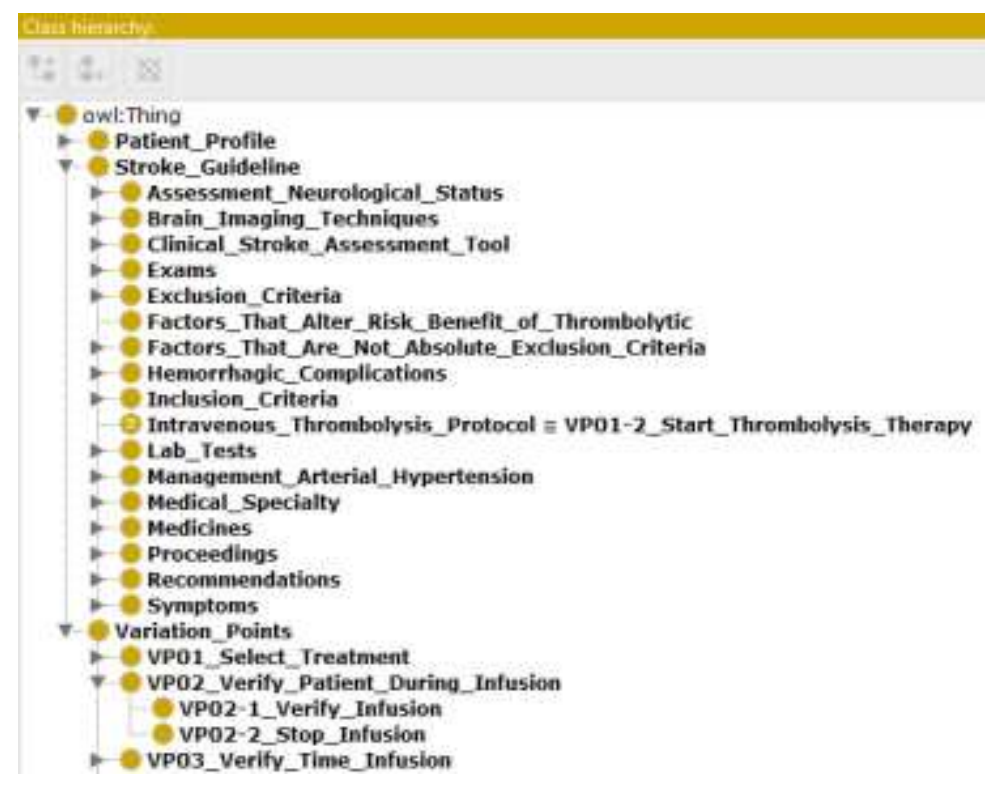

Figure 15 - Merged ontology for customizing the process model

According to Figure 15, the new ontology contains classes with the same label. This means that these classes must be verified. For equivalent classes, a new class is created. For example, the ontology contains two classes called 'Patient'. As result, a new class was created called 'Patient_Profile'. It is also noticeable that the ontology contains classes with the same meaning, but represented by different labels, such as Intravenous_Thrombolysis_Protocol and VP01-2_Start_Thrombolysis_Therapy. In this case, the classes are defined as equivalent. 
The SWRL rules can also be integrated after merging the ontologies. For example, a selection rule for decision point 'VP02-2_Stop_Infusion' in the process model customization ontology states that this decision point is only selected if the patient displays any signs of bleeding. The second ontology contains a set of rules defining the symptoms of hemorrhage, such as nausea, sudden increase of blood pressure, among others. In this way, both rules can be integrated. So that when one of the hemorrhage symptoms is selected, decision point VP02-2_Stop_Infusion is displayed for users. Additionally, according to the symptoms selected by users, recommendations can also be displayed for the user. Figure 16 shows that by answering the questionnaire and through the semantic reasoning, the next variation points and the relevant recommendations are displayed to users.

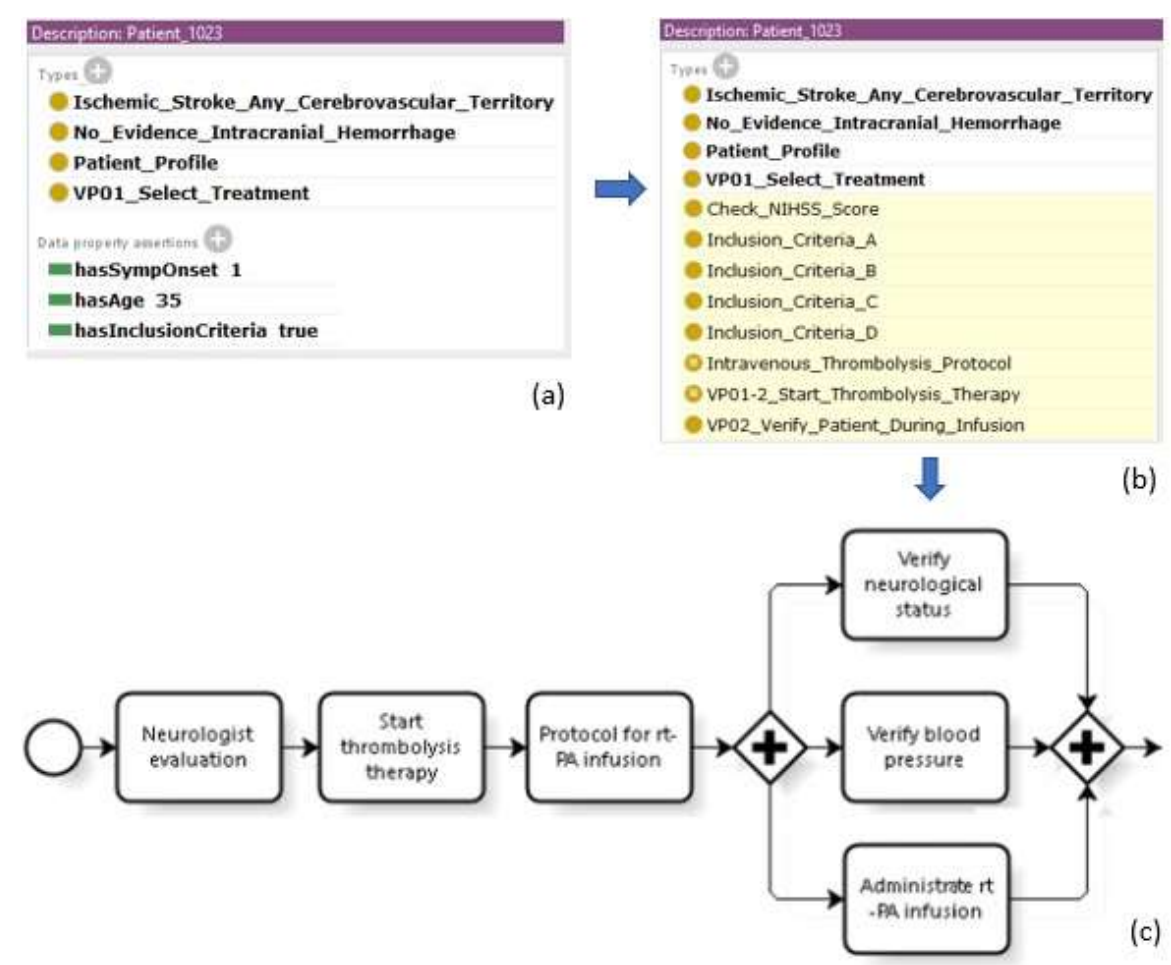

Figure 16 - Customizing the process model by reasoning on the merged ontology

Figure 16(a) shows the user-provided information about the patient with ID 1023, such as the patient's age, symptom onset and symptoms displayed. Figure 16(b) shows 
the ontology after the logic step. Given that the patient only displays inclusion criteria, the next activities are 'VP01-2_Start_Thrombolysis_Therapy' and VP02_Verify_Patient_During_Infusion. Treatment-related recommendations can also be displayed to users. For example, logic step in Figure 16(b) shows checking the patient's NIHSS score as recommendation. The semantic logic enables building process model. Figure 16(c) shows the fragment of the process model that shows that the patient can begin thrombolysis therapy.

Figure 17(a) shows that question 33, refers to identifying whether, during thrombolysis therapy, patients display any specific symptoms. If patients do not display any hemorrhage symptoms, users must select data property 'hasNoBleedingSymptoms' as True (according to Figure 17(b)). Then, users must inform how long the patient received infusion (Q34). Figure 17(b) shows the infusion time selected by the user as 90 (minutes). Thus, after the semantic reasoning (Figure 17(c)), the next activity is presented to users ('VP03-1_End_Infusion'). This means that the thrombolysis therapy was completely successful, and that the patient must now be monitored. In this way, the process variant can be customized as shown in Figure 17(d). 


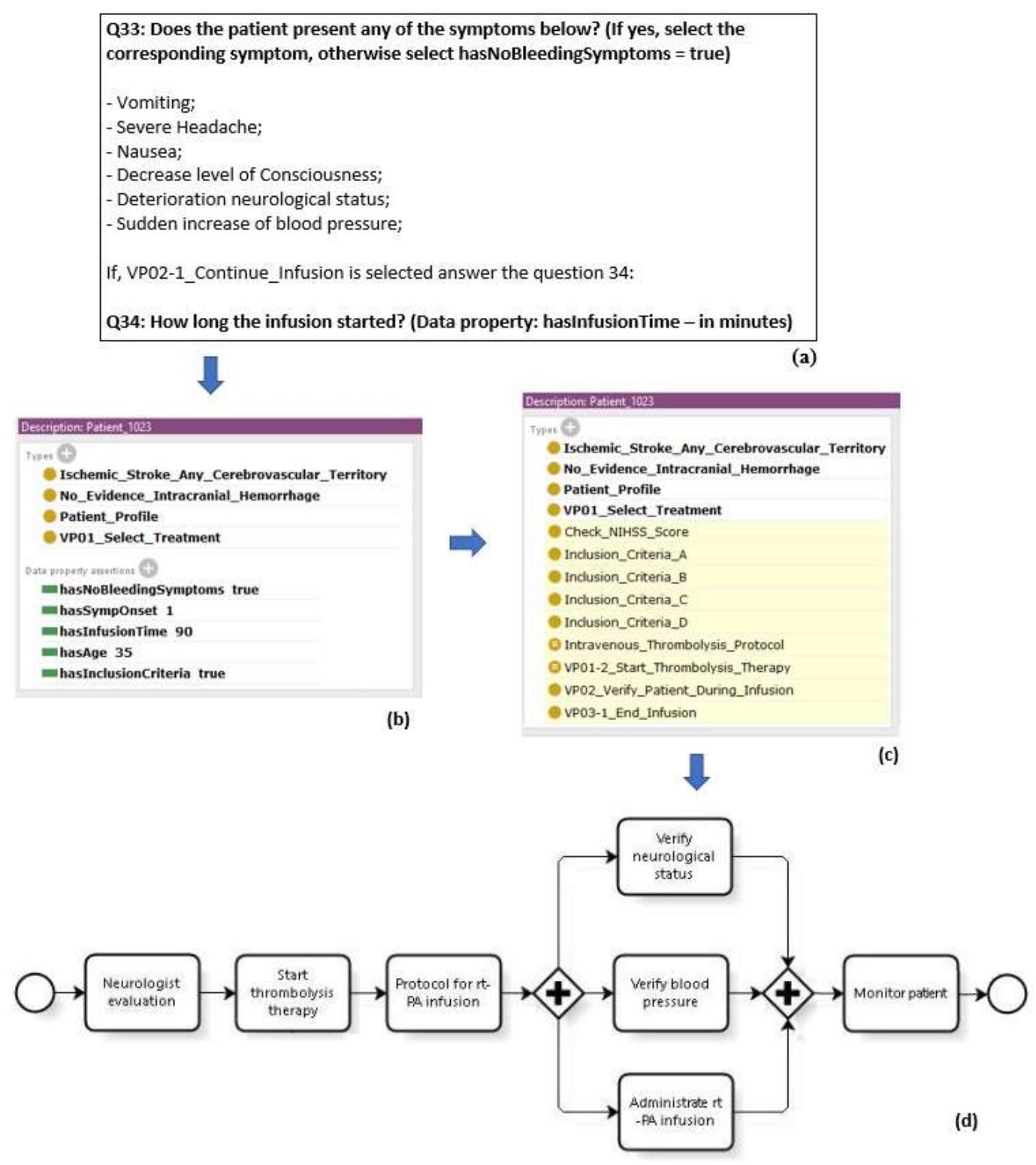

Figure 17 - Fragment of the resulting process variant after semantic reasoning

\section{Discussion and Conclusions}

Analysis of the existing approaches for customizing process models enables identifying some gaps, such as the need for information with respect to aspects for customizing the process model and the therapeutic context. There is also a need to display to users the expected impact of every selection made while customizing the process model. In this way, this paper aims to address these gaps by developing an approach for customizing process models by providing decision-making support for users through relevant 
recommendations, provided during customization.

The approach proposed is comprised of three steps. In the first step, the decision miner algorithm is applied to build the customizable process model. This algorithm enables discovering variants as well rules for selecting each one. The second step refers to developing the questionnaire that guides the users when customizing process models. In the last step, ontologies are applied for customizing the process model. By merging ontologies, the relevant knowledge can be used for process model customization. Therefore, as users select an alternative in the questionnaire and by applying semantic reasoning, they receive recommendations to drive selecting the appropriate process variant and, thus, improve the quality of the process.

This paper presents a case study that applies the approach proposed in acute ischemic stroke treatment. However, the proposed method can be used in any type of treatment characterized by a large amount of information, decision criteria, restrictions, and so on. The selection of the adequate treatment depends on many aspects, resulting in existence of several process variants. Therefore, by considering the choices made by users, the method can provide recommendations about the appropriate treatment for individual patients. Additionally, the method provides recommendations about the best practices for the patients' treatment considering clinical guidelines. As drawback, it is necessary to implement an execution engine that processes the resulting BPMN model, thus driving the process. However, as benefit this approach can help to decrease errors during the patient's treatment through the recommendations provided for the user.

According Bilici, Despotou and Arvanitis (2018), approaches for developing clinical decision support systems include decision rule models (e.g., Karadimas, Ebrahiminia, and Lepage, 2015), documentary models (e.g., Shiffman et al., 2001) or process-flow models also known as task-network models (TNMs). Regarding the TNMs 
models, some of the most known systems are: Guideline Interchange Format version 3 (GLIF3) (Peleg, Boxwala, Ogunyemi, et al., 2000) aims to create standards in health care enabling institutions and information systems to share the guidelines; Asbru (Shahar, Miksch, and Johnson, 1998), which consist of skeletal plans that represent the guidelines, but provides flexibility for executing specific activities; EON (Tu and Musen, 1999) provides recommendations based on the formalized clinical guideline and the patient's information; GUIDE (Quaglini et al., 2001) integrates the clinical guidelines into organizational workflows and applies Petri Nets to test and optimize the workflow model. When comparing these systems with the proposed approach, it is possible to note that the different systems show to the user the current position in a clinical guideline, but the proposed approach gives a step forward by providing to the user a view about the possible future activities that could be performed based on the information provided.

The approach presented in this paper shows that an ontology can be used to ensures that the rules for selecting each option in the variation points respects the recommendations from the clinical guidelines and expert knowledge. The next step refers to the integration of the questionnaire, ontology and the customizable process model, as well the automation of the approach, which allows to evaluate the approach. Besides, there are other perspectives that must be considered, such as hospital rules. In addition, all treatments provided for patients with acute ischemic stroke should be addressed using the approach proposed. As future work, the ontology concepts can be connected to elements in the process model generating the conditions needed for a process model customizing interface.

Investigating mining algorithms that enable the discovery of the rules linked to alternatives at decision points and how to better integrate them in the process mining techniques and simulation is also necessary. In addition, a more complete event log 
should be obtained for applying the approach proposed. The selection of the appropriate treatment regarding the acute ischemic stroke has some particularities, which are not consider in other types of treatments, such as the time of onset of symptoms. Thus, another issue refers to apply the proposed method in other type of treatment, such as the breast cancer.

\section{Disclosure statement}

No potential conflict of interest was reported by the authors.

\section{Funding}

This project is partially supported by Science Without Borders, CAPES, Brazil.

\section{ORCID}

\section{References}

Abburu, S. 2012. “A survey on ontology reasoners and comparison.” International Journal of Computer Applications, 57(17): 33-39. doi: 10.5120/9208-3748

Agrawal, G.L., and Gupta, H. 2013. "Optimization of C4. A decision tree algorithm for data mining application.” International Journal of Emerging Technology and Advanced engineering, 3(3): 341-345. doi: 10.1080/08839514.2018.1447479

Aized, T. 2009. "Modelling and performance maximization of an integrated automated guided vehicle system using coloured Petri net and response surface methods." Computers \& Industrial Engineering, 57(3): 822-831. doi: https://doi.org/10.1016/j.cie.2009.02.009

Andrew, A.M. 2004. "Ontologies: A Silver Bullet for Knowledge Management and Electronic Commerce." Kybernetes, 33 (9/10): 1544-1546, https://doi.org/10.1108/03684920410556142 
Asadi, M., Mohabbati, B., Gröner, G., and Gasevic, D. 2014. "Development and validation of customized process models." Journal of Systems and Software, 96: 73-92. doi: https://doi.org/10.1016/j.jss.2014.05.063

Ayora C., Torres V., Reichert M., Weber B., and Pelechano V. 2013a. "Towards RunTime Flexibility for Process Families: Open Issues and Research Challenges.” In: La Rosa M., Soffer P. (eds) Business Process Management Workshops. BPM 2012. Lecture Notes in Business Information Processing, vol. 132: 477-488. Springer, Berlin, Heidelberg. doi: https://doi.org/10.1007/978-3-642-36285-9_49

Ayora C., Torres V., Weber B., Reichert M., and Pelechano V. 2013b. "Enhancing Modeling and Change Support for Process Families through Change Patterns.” In: Nurcan S. et al. (eds) Enterprise, Business-Process and Information Systems Modeling. BPMDS 2013, EMMSAD 2013. Lecture Notes in Business Information Processing, vol. 147: 246-260. Springer, Berlin, Heidelberg. doi: https://doi.org/10.1007/978-3-642-38484-4_18

Ayora, C., Torres, V., Weber, B., Reichert, M., and Pelechano, V. 2015. "VIVACE: A framework for the systematic evaluation of variability support in process-aware information systems." Information and Software Technology, 57: 248-276. doi: https://doi.org/10.1016/j.infsof.2014.05.009

Beimel, D., and Peleg, M. 2011. "Using OWL and SWRL to represent and reason with situation-based access control policies.” Data \& Knowledge Engineering, 70(6): 596-615. doi: https://doi.org/10.1016/i.datak.2011.03.006

Bilici, E., Despotou, G., and Arvanitis, T.N. 2018. The use of computer-interpretable clinical guidelines to manage care complexities of patients with multimorbid conditions: A review. Digital health, 4, 2055207618804927.

Bose, R.J.C., and Van Der Aalst, W.M.P. 2012. "Process diagnostics using trace alignment: opportunities, issues, and challenges." Information Systems, 37(2): 117-141. doi: https://doi.org/10.1016/j.is.2011.08.003

Buijs J.C.A.M., van Dongen B.F., and van der Aalst W.M.P. 2013. "Mining Configurable Process Models from Collections of Event Logs.” In: Daniel F., Wang J., Weber B. (eds) Business Process Management. BPM 2013. Lecture Notes in Computer Science, vol. 8094: 33-48. Springer, Berlin, Heidelberg. doi: https://doi.org/10.1007/978-3-642-40176-3_5

Buijs J.C.A.M., and Reijers H.A. 2014. "Comparing Business Process Variants Using Models and Event Logs”. In: Bider I. et al. (eds) Enterprise, Business-Process and 
Information Systems Modeling. BPMDS 2014, EMMSAD 2014. Lecture Notes in Business Information Processing, vol. 175: 154-168. Springer, Berlin, Heidelberg. doi: https://doi.org/10.1007/978-3-662-43745-2_11

Bühne, S., Halmans, G. \& Pohl, K. Modelling dependencies between variation points in use case diagrams. Proceedings of 9th Intl. Workshop on Requirements Engineering-Foundations for Software Quality (pp. 59-70), 2003.

Cai, H., Xu, L., Xu, B., Zhang, P., Guo, J., \& Zhang, Y. (2018). A service governance mechanism based on process mining for cloud-based applications. Enterprise Information Systems, 1-18.

CPN Tools. Accessed March 25 2017. http://cpntools.org/.

Corcho, O., Fernández-López, M., and Gómez-Pérez, A. 2007. “Ontological engineering: what are ontologies and how can we build them?" Chapter 3 in Semantic Web Services: Theory, Tools and Applications, pp. 44-70.

De Medeiros, A.A., and Günther, C.W. 2005. "Process mining: Using CPN tools to create test $\log$ s for mining algorithms." Proceedings of the sixth workshop on the practical use of coloured Petri nets and CPN tools (CPN 2005) (pp. 177-190). (DAIMI; Vol. 576). Aarhus, Denmark: University of Aarhus.

De Medeiros, A.K.A, Pedrinaci, C., Van Der Aalst, W.M.P., Domingue, J., Song, M., Rozinat, A., Norton, B., and Cabral, L. 2007. “An Outlook on Semantic Business Process Mining and Monitoring.” In: Meersman R., Tari Z., Herrero P. (eds) On the Move to Meaningful Internet Systems 2007: OTM 2007 Workshops. OTM 2007. Lecture Notes in Computer Science, vol 4806. Springer, Berlin, Heidelberg. doi: https://doi.org/10.1007/978-3-540-76890-6_52

Detro, S.P., Morozov, D., Lezoche, M., Panetto, H., Santos, E.A.P., and Zdravkovic, M. 2016. "Enhancing semantic interoperability in healthcare using semantic process mining." 6th International Conference on Information Society and Techology, ICIST 2016, Feb 2016, Kopaonik, Serbia. 1, pp.80-85, 2016. <hal-01298125>

Djellali, C. 2013. "A new data mining system for ontology learning using dynamic time warping alignment as a case." Procedia Computer Science, (21): 75-82. doi: https://doi.org/10.1016/j.procs.2013.09.012

El Faquih, L., Sbai, H., and Fredj, M. 2014. "Semantic variability modeling in business processes: A comparative study.” The 9th International Conference for Internet Technology and Secured Transactions (ICITST-2014) (pp. 131-136). doi: $\underline{10.1109 / I C I T S T .2014 .7038792}$ 
El Faquih, L., Sbai, H., Fredj, M. 2015. "Configurable process models: A semantic validation." 2015 10th International Conference on Intelligent Systems: Theories and Applications (SITA) (pp. 1-6). doi: 10.1109/SITA.2015.7358436

Fernández-Llatas, C., Benedi, J.M., García-Gómez, J.M., and Traver, V. 2013. "Process mining for individualized behavior modeling using wireless tracking in nursing homes." Sensors, 13(11):15434-15451. doi: 10.3390/s131115434

Gašević, D., Djuric, D., and Devedžic, V. 2009. Model driven engineering and ontology development. Springer-Verlag Berlin Heidelberg. doi: 10.1007/978-3-64200282-3

Gottschalk F., Wagemakers T.A.C., Jansen-Vullers M.H., van der Aalst W.M.P., and La Rosa M. 2009. "Configurable Process Models: Experiences from a Municipality Case Study.” In: van Eck P., Gordijn J., Wieringa R. (eds) Advanced Information Systems Engineering. CAiSE 2009. Lecture Notes in Computer Science, vol 5565: 486-500. Springer, Berlin, Heidelberg. doi: https://doi.org/10.1007/978-3642-02144-2_38

Gruber, T.R. 1995. "Toward principles for the design of ontologies used for knowledge sharing?” International Journal of Human-Computer Studies, vol. 43 (5-6): 907928. doi: https://doi.org/10.1006/ijhc.1995.1081

Günther, C., Rozinat, A., Van Der Aalst, W.M.P., and Van Uden, K. 2008. "Monitoring deployed application usage with process mining." BPM Center Report BPM-0811 pp., 1-8, 2008.

Haav, H.M. 2004. "A Semi-automatic Method to Ontology Design by Using FCA.” In CLA. Available in: http://ftp.informatik.rwth-aachen.de/Publications/CEUR-WS/Vol110/paper2.pdf

Hallerbach, A., Bauer, T., and Reichert, M. 2010a. "Capturing variability in business process models: the Provop approach.” Journal of Software Maintenance and Evolution: Research and Practice, 22(6-7): 519-546. doi: 10.1002/smr.v22:6/7

Hallerbach, A., Bauer, T., and Reichert, M. 2010b. "Configuration and management of process variants." In: Brocke J.., Rosemann M. (eds) Handbook on Business Process Management 1. International Handbooks on Information Systems. Springer, Berlin, Heidelberg. doi: https://doi.org/10.1007/978-3-642-00416-2_11

Hoang, H.H., Jung, J.J., \& Tran, C.P. 2014. Ontology-based approaches for crossenterprise collaboration: a literature review on semantic business process management. Enterprise Information Systems, 8(6), 648-664. 
Huang, Y., Feng, Z., He, K., and Huang, Y. 2013. "Ontology-based configuration for service-based business process model." 2013 IEEE International Conference on Services Computing (2013): 296-303. doi: $\underline{10.1109 / \text { SCC.2013.59 }}$

Ingvaldsen, J.E., \& Gulla, J.A. 2012. Industrial application of semantic process mining. Enterprise Information Systems, 6(2), 139-163.

Jans, M., Van Der Werf, J., Lybaert, N., and Vanhoof, K. 2011. "A business process mining application for internal transaction fraud mitigation." Expert Systems with $\begin{array}{lll}\text { applications, } & \text { 38(10): } & \text { 13351-13359. }\end{array}$ https://doi.org/10.1016/j.eswa.2011.04.159

Kalibatiene, D., and Vasilecas, O. 2011. "Survey on ontology languages.” In: Grabis J., Kirikova M. (eds) Perspectives in Business Informatics Research. BIR 2011. Lecture Notes in Business Information Processing, vol 90. Springer, Berlin, Heidelberg. doi: https://doi.org/10.1007/978-3-642-24511-4_10

Karadimas, H., Ebrahiminia, V., Lepage, E. 2015. User-defined functions in the Arden Syntax: An extension proposal. Artif Intell Med. doi: 10.1016/j.artmed.2015.11.003.

Jensen, K., Kristensen, L.M., and Wells, L. 2007. "Coloured Petri Nets and CPN Tools for modelling and validation of concurrent systems." International Journal on Software Tools for Technology Transfer, 9(3-4): 213-254. doi: https://doi.org/10.1007/s10009-007-0038-X

La Rosa, M., Dumas, M., and Ter Hofstede, A.H. 2009. "Modelling Business Process Variability for Design-Time Configuration." Chapter 9 in Cardoso, Jorge \& van der Aalst, Wil M.P. (Eds.) Handbook of Research on Business Process Modeling. Information Science Reference (IGI Global), Hershey, PA, pp. 204-228. doi: 10.4018/978-1-60566-288-6.ch009

La Rosa, M., Van Der Aalst, W.M.P., Dumas, M., and Milani, F.P. 2017. "Business process variability modelling: A survey.” ACM Computing Surveys, 50(1), 2:12:45. doi: $\underline{10.1145 / 3041957}$

Li, C., Reichert, M., and Wombacher, A. 2008a. "Discovering reference process models by mining process variants." 2008 IEEE International Conference on Web Services. doi: $\underline{10.1109 / \text { ICWS.2008.13 }}$

Li, C., Reichert, M., and Wombacher, A. 2008b. Mining based on learning from process change logs. In: Ardagna D., Mecella M., Yang J. (eds) Business Process Management Workshops. BPM 2008. Lecture Notes in Business Information 
Processing, vol 17. Springer, Berlin, Heidelberg. doi: https://doi.org/10.1007/9783-642-00328-8_12

Li, C., Reichert, M., and Wombacher, A. 2010. "The MinAdept clustering approach for discovering reference process models out of process variants." International Journal of Cooperative Information Systems, 19(3-4): 159-203. doi: $\underline{10.1142 / \mathrm{S} 0218843010002139}$

Li, J., Yang, J. J., Liu, C., Zhao, Y., Liu, B., \& Shi, Y. 2014. Exploiting semantic linkages among multiple sources for semantic information retrieval. Enterprise Information Systems, 8(4), 464-489.

Luo, J., Meng, B., Quan, C., \& Tu, X. 2016. Exploiting salient semantic analysis for information retrieval. Enterprise Information Systems, 10(9), 959-969.

Mans, R., Reijers, H., Berends, H., Bandara, W., and Rogier, P. 2013. "Business process mining success.” Proceedings of the 21st European Conference on Information Systems. AIS Electronic Library (AISeL).

Martins, S.C.O., Freitas, G.R.D., Pontes-Neto, O.M., Pieri, A., Moro, C.H.C., Jesus, P.A.P.D., Longo, A., Evaristo, E.F., Carvalho, J.J.F.D., Fernandes, J.G., and Gagliardi, R.J. 2012. "Guidelines for acute ischemic stroke treatment: part II: stroke treatment." Arquivos de neuro-psiquiatria, 70(11): 885-893.

Martinez-Gil, J. 2015. “Automated knowledge base management: A survey.” Computer Science Review, 18:1-9. doi; https://doi.org/10.1016/j.cosrev.2015.09.001

Menárguez-Tortosa, M., and Fernández-Breis, J.T. 2013. “OWL-based reasoning methods for validating archetypes." Journal of biomedical informatics, 46(2): 304-317. doi: https://doi.org/10.1016/j.jbi.2012.11.009

Noy, N.F., and Musen, M.A. 2000. “Algorithm and tool for automated ontology merging and alignment." Proceedings of the 17th National Conference on Artificial Intelligence (AAAI-00). Available as SMI technical report SMI-2000-0831, 2000.

Obitko, M. 2007. "Translations between ontologies in multi-agent systems." $\mathrm{Ph}$. D. Dissertation, Czech Technical University, Faculty of Electrical Engineering.

Oliveira-Filho, J. et al. 2012. "Guidelines for acute ischemic stroke treatment: part I." Arq. Neuro-Psiquiatr., São Paulo, 70(8): 621-629.

Pedrinaci, C., and Domingue, J. 2007. "Towards an ontology for process monitoring and mining.” CEUR Workshop Proceedings, vol. 251: 76-87.

Peleg, M., Boxwala, A. A., Ogunyemi, O., Zeng, Q., Tu, S., Lacson, R., ... and Shortliffe, E.H. 2000. GLIF3: the evolution of a guideline representation format. In 
Proceedings of the AMIA Symposium (p. 645). American Medical Informatics Association.

Pinto, H.S., Gómez-Pérez, A., and Martins, J.P. 1999. "Some issues on ontology integration." 16th International Joint Conference on Artificial Intelligence (IJCAI'99) Workshop: KRR5: Ontologies and Problem-Solving Methods: Lesson Learned and Future Trends.

ProM, 2018. Accessed 20 Jan 2018. http://www.promtools.org/doku.php.

ProM Import Framework, 2018. 01 Feb 2018. http://www.promtools.org/promimport/.

Protégé, 2017. 18 Oct 2017. https://protege.stanford.edu/

Quaglini, S., Stefanelli, M., Lanzola, G., Caporusso, V., Panzarasa, S. 2001. Flexible guideline-based patient careflow systems. Artif Intell Med, 22:65-80.

Quaglini, S. 2008. "Compliance with clinical practice guidelines." Chapter 9 in Computer-based Medical Guidelines and Protocols: A Primer and Current Trends, Technology and Informatics 139: 160-179.

Quinlan, J.R. 2014. “C4. 5: programs for machine learning.” Elsevier.

Reichert, M., and Weber, B. 2012. "Enabling flexibility in process-aware information systems: challenges, methods, technologies." Springer Science \& Business Media.

Rico, M., Caliusco, M.L., Chiotti, O., \& Galli, M.R. 2015. An approach to define semantics for BPM systems interoperability. Enterprise Information Systems, 9(3), 324-347.

Rozinat, A., Mans, R.S., and Van Der Aalst, W.M.P. 2006. "Mining CPN models: discovering process models with data from event logs". Workshop and Tutorial on Practical Use of Coloured Petri Nets and the CPN.

Rozinat, A., and Van Der Aalst, W.M.P. 2006. "Decision mining in business processes." (BETA publicatie: working papers; Vol. 164). Eindhoven: Technische Universiteit Eindhoven.

Rozinat, A., De Jong, I.S.M., Günther, C.W., and Van Der Aalst, W.M.P. 2009. "Process mining applied to the test process of wafer scanners in ASML." Systems, Man, and Cybernetics, Part C: Applications and Reviews. IEEE Transactions on vol. 39(4): 474-479.

Shahar, Y., Miksch, S., Johnson, P. 1998. The Asgaard Project: a task-specific Framework for the application and critiquing of time-oriented clinical guidelines. Artif Intell Med, 14:29-51. 
Sharman, R., Kishore, R., and Ramesh, R. 2007. "Ontologies: a handbook of principles, concepts and applications in information systems." Springer's Integrated Series in Information Systems.

Shiffman R.N., Agrawal A., Deshpande, A.M., et al. 2001. An approach to guideline implementation with GEM. Stud Health Technol Inform; 84(1): 271-275.

Song, F., Zacharewicz, G., and Chen, D. 2013. “An ontology-driven framework towards building enterprise semantic information layer." Advanced Engineering Informatics, 27(1): 38-50. doi: https://doi.org/10.1016/j.aei.2012.11.003

Taye, M.M. 2010. "The State of the Art: Ontology Web-Based Languages: XML Based." Journal of Computing, 2(6): 166-176.

Torres, V., Zugal, S., Weber, B., Reichert, M., Ayora, C., and Pelechano, V. 2013. “A qualitative comparison of approaches supporting business process variability.” In: La Rosa M., Soffer P. (eds) Business Process Management Workshops. BPM 2012. Lecture Notes in Business Information Processing, vol 132. Springer, Berlin, Heidelberg.

Tu, S.W., Musen, M.A. 1999. A flexible approach to guideline modeling. Proc AMIA Symp.

Valença, G., Alves, C., Alves, V., and Niu, N. 2013. “A systematic mapping study on business process variability." International Journal of Computer Science \& Information Technology, 5(1): 1:21.

Van Der Aalst, W.M.P., Dumas, M., Gottschalk, F., Ter Hofstede, A.H., La Rosa, M., and Mendling, J. 2008. "Correctness-preserving configuration of business process models." In: Fiadeiro J.L., Inverardi P. (eds) Fundamental Approaches to Software Engineering. FASE 2008. Lecture Notes in Computer Science, vol 4961. Springer, Berlin, Heidelberg. doi: https://doi.org/10.1007/978-3-540-78743-3_4

Van Der Aalst, W.M.P., Adriansyah, A., De Medeiros, A.K.A., Arcieri, F., Baier, T., Blickle, T., Bose, J.C., Van Den Brand, P., Brandtjen, R., Buijs, J., and Burattin, A. 2011. "Process mining manifesto.” In: Daniel F., Barkaoui K., Dustdar S. (eds) Business Process Management Workshops. BPM 2011. Lecture Notes in Business Information Processing, vol 99. Springer, Berlin, Heidelberg. doi: https://doi.org/10.1007/978-3-642-28108-2_19

Van Der Aalst, W.M.P. 2012. "Service Mining: Using Process Mining to Discover, Check, and Improve Service Behavior." IEEE Transactions on Service Computing, 6(4): 525:535. doi: 10.1109/TSC.2012.25 
Van Der Aalst, W.M.P., and Dustdar, S. 2012. Process mining put into context. IEEE Internet Computing, 16(1): 82-86. doi: 10.1109/MIC.2012.12

Weske, M. 2012. Business process management: concepts, languages, architectures. Springer-Verlag Berlin Heidelberg. doi: 10.1007/978-3-642-28616-2

World Stroke Organization, 2017. Accessed 17 Oct 2017. http://www.world-stroke.org/ 